\title{
openheart Randomised comparison of zofenopril and ramipril plus acetylsalicylic acid in postmyocardial infarction patients with left ventricular systolic dysfunction: a post hoc analysis of the SMILE-4 Study in patients according to levels of left ventricular ejection fraction at entry
}

To cite: Borghi C, Omboni S, Cicero AFG, et al.

Randomised comparison of zofenopril and ramipril plus acetylsalicylic acid in postmyocardial infarction patients with left ventricular systolic dysfunction: a post hoc analysis of the SMILE-4 Study in patients according to levels of left ventricular ejection fraction at entry. Open Heart 2015;2:e000195 doi:10.1136/openhrt-2014000195

Received 16 September 2014 Revised 22 December 2014 Accepted 23 June 2015

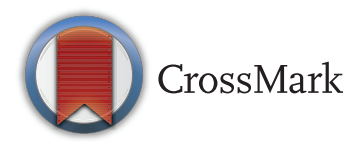

For numbered affiliations see end of article.

Correspondence to Professor Claudio Borghi; claudio.borghi@unibo.it

\section{ABSTRACT}

Objective: Conflicting evidence exists on the benefits of treating patients with coronary artery disease and preserved left ventricular ejection fraction (LVEF) with an ACE inhibitor. This retrospective analysis of the SMILE-4 Study sought to compare the efficacy of zofenopril $60 \mathrm{mg}$ plus acetylsalicylic acid (ASA) versus ramipril $10 \mathrm{mg}$ plus ASA $100 \mathrm{mg}$ in patients with acute myocardial infarction (AMI) and heart failure, according to an impaired or preserved LVEF.

Methods: The primary study end point was 1-year combined occurrence of death or hospitalisation for cardiovascular causes. A preserved LVEF was defined by a baseline LVEF $>40 \%$ and an impaired one by an LVEF $\leq 40 \%$.

Results: 448 patients (63\%) had preserved and 262 $(37 \%)$ had impaired LVEF. The primary end point occurred in 125 patients with preserved (28\%) and 106 patients with impaired LVEF $(41 \%, p=0.001)$. In the first group, the rate of major cardiovascular events was significantly lower under zofenopril than under ramipril (23\% vs $33 \%$; OR and $95 \% \mathrm{Cl} 0.60,0.39$ to 0.91 ; $\mathrm{p}=0.016$ ). This was also the case for patients with impaired LVEF, though between-group difference was not statistically significant ( $38 \%$ zofenopril vs $44 \%$ ramipril; OR $0.77,0.47$ to $1.26 ; p=0.297$ ). LVEF values significantly $(p<0.0001)$ increased during the follow-up in both subsets with no between-treatment differences. However, improvement rates in LVEF (increase $\geq 5 \%$ ) were higher in patients with impaired LVEF (72\% vs $61 \%, \mathrm{p}=0.006)$.

Conclusions: In the SMILE-4 Study, the cardiovascular outcome of patients with post-AMI with preserved LVEF was more favourable in the zofenopril than in the ramipril treatment group.

Trial registration number: EudraCT Number: 2004001150-88 (http://www.clinicaltrialsregister.eu); Italian Ministry of Health Code: GUIDOTT_III_2004_001 (https:// oss-sper-clin.agenziafarmaco.it).

\section{KEY MESSAGES}

What is already known about this subject?

- Guidelines state that it is reasonable to prescribe ACE inhibitors not only to patients who have coronary artery disease and either signs of heart failure or impaired systolic function, but also to patients with stable or unstable ischaemic heart disease and preserved left ventricular ejection fraction (LVEF), with well-controlled cardiovascular risk factors. However, few studies have been conducted in order to evaluate the effectiveness of ACE inhibitors in such a category of patients; and the cumulative evidence provided by meta-analyses of such studies shows only a modest favourable effect of ACE inhibitors on patient outcome.

What does this study add?

- This study shows that patients with coronary artery disease and preserved LVEF may have a long-term benefit from treatment with an ACE inhibitor. However, the response to treatment in terms of prevention of major cardiovascular outcomes is better with a sulfhydryl-containing ACE inhibitor, such as zofenopril, than with a non-sulfhydryl-containing ACE inhibitor, such as ramipril.

How might this impact on clinical practice?

- The study provides further support for the treatment of a wide range of patients following an acute myocardial infarction (AMI) with an effective ACE inhibitor and demonstrates that patients with post-AMI with preserved LVEF deserve long-term treatment with an ACE inhibitor. The ACE inhibitor should be chosen carefully among those proved to be effective in these patients. 


\section{INTRODUCTION}

Large randomised, placebo-controlled trials clearly demonstrated that ACE inhibitors are beneficial for patients who have coronary artery disease and either signs of heart failure (HF) or impaired systolic function. ${ }^{1}$ Systematic overviews of trials of ACE inhibition early in ST-segment elevated acute myocardial infarction (AMI) indicate that this therapy is safe, well tolerated and associated with a small but significant reduction in 30-day mortality. ${ }^{2}$ Based on such evidence, current guidelines for the management of patients with AMI recommend the prescription of an ACE inhibitor to all patients with ST-segment elevation AMI (STEMI) and an impaired left ventricular ejection fraction (LVEF $<40 \%$ ), or those who have experienced HF in the early phase. ${ }^{3} 4$

Guidelines also state that it is reasonable to prescribe ACE inhibitors for patients with stable or unstable ischaemic heart disease and preserved LVEF, with wellcontrolled cardiovascular risk factors. ${ }^{5}{ }^{6}$ Nonetheless, few studies have been conducted in order to evaluate the use of ACE inhibitors in patients who have ischaemic heart disease, but without clinical evidence of $\mathrm{HF}$ or frank left ventricular dysfunction (LVD). The cumulative evidence provided by meta-analyses of such studies shows only a modest favourable effect of ACE inhibitors on the outcome of such patients. ${ }^{7-9}$

In order to gain better insight into this controversy, and to determine whether, and to what extent, longterm prescription of two pharmacologically different ACE inhibitors may decrease the risk of major cardiovascular events and mortality in patients who have coronary artery disease and preserved LVEF, we undertook a post hoc analysis of the SMILE-4 Study. ${ }^{10}$ This direct comparative trial demonstrated that in patients with post-AMI with LVD, the efficacy of zofenopril associated with acetylsalicylic acid (ASA) was superior to that of ramipril plus ASA, in terms of prevention of major cardiovascular outcomes. ${ }^{10}$ In the present post hoc analysis, we evaluated the prognostic benefit of the two ACE inhibitors in the two subsets of participants with preserved and impaired LVEF.

\section{METHODS}

\section{Study design}

Details on the methodology and main results of the SMILE-4 Study are reported elsewhere. ${ }^{10}$ In brief, this multicentre, multinational, randomised, prospective study, was conducted at 79 hospitals in eight different European countries and coordinated by the Internal Medicine Unit of the University of Bologna (Italy). The study enrolled male and non-pregnant female patients, aged 18-85 years, with a confirmed diagnosis of STEMI or NSTEMI (non-ST-segment elevation AMI) in the $24 \mathrm{~h}$ preceding the enrolment (not treated with primary percutaneous transluminal angioplasty or PTCA, treated or not with thrombolysis and recommended pharmacological treatment) and with clinical and/or echocardiographic evidence of LVD (Killip class $>1$, plus third heart sound or pulmonary congestion on chest X-ray and/or an LVEF, <45\%).

Initially, all the eligible patients entered a 4-day openlabel phase when zofenopril was administered according to an up-titration scheme. On days 1 and 2, patients received zofenopril $7.5 \mathrm{mg}$ twice daily plus an evening dose of ASA $100 \mathrm{mg}$. On days 3 and 4, the zofenopril dose was doubled (15 mg twice daily) while that of ASA remained unchanged. This choice was based on ethical and regulatory reasons, and justified by previous evidence of efficacy and safety of the early zofenopril treatment in patients with anterior AMI. ${ }^{11}$ On day 5 , patients were randomised 1:1 double-blind (using a centralised, computer-generated randomisation list) to receive zofenopril $30 \mathrm{mg}$ twice daily plus ASA $100 \mathrm{mg}$ once daily, or ramipril $5 \mathrm{mg}$ twice daily plus ASA $100 \mathrm{mg}$ once daily for 12 months. The study medications were administered in combination with standard recommended treatments for AMI, excluding other ACE inhibitors, angiotensin receptor blockers (ARBs) and antiplatelet drugs other than ASA, clopidogrel or ticlopidine. Concomitant chronic anticoagulant treatment was allowed in the acute phase of myocardial infarction (MI) and in case of a specific indication or in patients who reached a study end point. The first patient was enrolled on March 2005, and the last patient completed on July 2009. Patients were seen at enrolment, at randomisation (5-days after enrolment) and after 1, 6 and 12 months. At the initial and at any subsequent visit, blood pressure was measured, an echocardiogram was performed, blood samples were drawn (centralised estimation of $\mathrm{N}$ terminal pro-brain natriuretic peptide (NT-proBNP)) and occurrence of concomitant diseases, adverse events, use of concomitant medications and compliance to study drugs were checked. A physical examination, a 12-lead ECG and laboratory tests (haematology, clinical chemistry and urinalysis) were performed at entry, at randomisation and at study end.

\section{Echocardiography}

All patients underwent a standard echocardiography examination, including determination of LVEF and left ventricular systolic and diastolic volumes, as recommended by the American Society of Echocardiography. ${ }^{12}$ All the measurements were performed by expert sonographers and read at the local site.

A two-dimensional examination was performed in each participant while lying in a left lateral decubitus position. To obtain volumetric measurements, the apical four-chamber and two-chamber views and the biplane Simpson's rule, requiring manual tracing of the left ventricular endocardial border in end-diastole and endsystole, were used. For each view, at least three consecutive cardiac cycles were recorded and measurement reflected the average of these three cardiac cycles. To minimise cardiac movements resulting from respiration, all echocardiographic data were obtained at 
end-expiration. LVEF was calculated in the standard fashion from left ventricular end-diastolic volume and left ventricular end-systolic volume.

\section{Statistical analysis}

This retrospective analysis was based on the comparison of the original study primary end point between zofenopril-treated and ramipril-treated patients with preserved LVEF $(>40 \%)$ and impaired LVEF $(\leq 40 \%)$. This cut-off was selected because in these patients, the largest evidence of effectiveness is available to date from major trials. ${ }^{13}$ However, since recent trials enrolled patients with preserved LVEF, presenting without HF and with an LVEF $>50 \%$, an additional analysis was run by applying a $50 \%$ cut-off. ${ }^{14} 15$ The primary study end point was the 1-year combined occurrence of cardiovascular mortality or hospitalisation because of cardiovascular causes (congestive HF, AMI, angina or a decline in LVEF >15\%). Secondary study end points included hospitalisation because of, and death from, cardiovascular causes.

Data analysis was carried out on the intention-to-treat population, defined as patients treated with at least one dose of study medication and documenting at least once the measure of the primary efficacy assessment, even in case of protocol violation or premature withdrawal from the study.

The baseline characteristics and the distribution of variables in patients with preserved and impaired LVEF, and separately, in the zofenopril and ramipril populations, were compared using a $\chi^{2}$ test (categorical variables) and $\mathrm{a} t \mathrm{t}$ test (continuous variables). The difference between treatment groups with respect to the study end points was assessed by calculating the estimated OR and the corresponding 95\% CI. A logistic regression analysis was used for the comparison between the two treatment groups, by adjusting for the prevalence of PTCA at entry. Survival curves were drawn using Kaplan-Meier estimates and a survival analysis was performed according to the log-rank statistics. An interaction test, based on logistic regression analysis, was applied to test difference for the OR between the two study subgroups (preserved vs impaired LVEF) and the two study treatments (zofenopril vs ramipril) for the primary study end point.

All $\mathrm{p}$ values are two-tailed and a $\mathrm{p}$ value $<0.05$ is considered as an indication of statistical significance.

\section{RESULTS}

\section{Study population}

Information on LVEF at baseline was available for 710 of the 716 patients of the intention-to-treat population. Overall, $448(63 \%)$ patients were classified as having a preserved LVEF and $262(37 \%)$ as having an impaired LVEF at baseline. Baseline characteristics of these two subgroups of participants are presented in table 1. On average, patients with a preserved LVEF were younger and were less likely to report a previous diagnosis of diabetes, MI, angina pectoris or congestive HF. Statistically significant differences between the two groups were also observed in infarct location, with a lower prevalence in anterior infarct in patients with a preserved LVEF, in the frequency of PTCA at entry (more common among patients with preserved LVEF), in the level of estimated glomerular filtration rate (GFR) (higher in the group with preserved LVEF) and in NT-proBNP and heart rate values (lower in patients with preserved LVEF).

Treatment distribution was well balanced within the two groups. Among patients with preserved LVEF, 213 were administered zofenopril (48\%) and 235 ramipril (53\%); for the group of patients with impaired LVEF, the corresponding figures were $151(58 \%)$ and 111 (42\%), respectively. Within the two subgroups with preserved or impaired LVEF, the sets of patients treated with zofenopril or ramipril were comparable for baseline characteristics. The only significant difference was observed in the group with preserved LVEF and was represented by a larger $(6 \%$ vs $1 \%, \mathrm{p}=0.018)$ prevalence of patients with a history of congestive $\mathrm{HF}$ and a lower (3\% vs $7 \%, \mathrm{p}=0.047)$ prevalence of patients with a previous PTCA in the ramipril-treated group, and lower (86 \pm 31 vs $93 \pm 37 \mathrm{~mL} / \mathrm{min}, \mathrm{p}=0.044$ ) levels of estimated GFR in zofenopril-treated patients. In both the preserved and impaired LVEF subgroups, no difference was observed between the two treatment groups in the rate of patients with PTCA or thrombolysis at entry.

\section{Primary outcome measure}

During the 12 months of follow-up, cardiovascular death or hospitalisation occurred in 125 patients with preserved LVEF (28\%) and in 106 patients with impaired LVEF $(41 \%, \mathrm{p}=0.001)$. In the group with preserved LVEF, the primary end point occurred in $23 \%$ of zofenopril-treated patients and in 33\% of ramipriltreated patients (table 2). This resulted in a $41 \%$ significantly $(\mathrm{p}=0.015$ logistic regression analysis and $\mathrm{p}=0.021$ log-rank test) lower risk of achieving the combined end point (figure 1). In the group with impaired LVEF, the combined primary end point was reported in $38 \%$ of zofenopril-treated patients and in $44 \%$ of ramipriltreated patients, with no between-group statistically significant differences $(\mathrm{p}=0.403$ logistic regression analysis and $\mathrm{p}=0.436$ log-rank test) (table 2 and figure 1 ).

When the $50 \%$ LVEF cut-off was used, 83 patients had a preserved LVEF and 627 an impaired LVEF. In the subgroup with preserved LVEF, 10 events were reported in 40 zofenopril-treated patients $(25 \%)$ and 16 in 43 ramipril-treated patients (37\%): the OR was similar to that observed when patients were classified according to a $40 \%$ LVEF cut-off (0.56 (0.22 to 1.45), $\mathrm{p}=0.231$ ). A major cardiovascular event occurred in 95 of 324 patients $(29 \%)$ with an impaired LVEF $(\leq 50 \%)$ treated with zofenopril and in 110 of 303 treated with ramipril (36\%), with an OR of 0.73 (0.52 to 1.02$)$ ( $\mathrm{p}=0.063$ ). 
Table 1 Baseline demographic characteristics of the patients with a preserved LVEF $>40 \%$ and with an impaired LVEF $(\leq 40 \%)$

\begin{tabular}{|c|c|c|c|}
\hline & Preserved LVEF $(n=448)$ & Impaired LVEF $(n=262)$ & p Value \\
\hline Age (years, mean $\pm S D$ ) & $59.7 \pm 10.9$ & $62.8 \pm 10.4$ & $<0.001$ \\
\hline \multicolumn{4}{|l|}{ Gender $(n, \%)$} \\
\hline Male & $345(77.0)$ & $194(74.0)$ & \multirow[t]{2}{*}{0.373} \\
\hline Female & $103(23.0)$ & $68(26.0)$ & \\
\hline $\mathrm{BMI}\left(\mathrm{kg} / \mathrm{m}^{2}\right.$, mean $\left.\pm \mathrm{SD}\right)$ & $27.7 \pm 3.7$ & $27.8 \pm 4.3$ & 0.663 \\
\hline \multicolumn{4}{|l|}{ History of } \\
\hline Diabetes mellitus $(n, \%)$ & $64(14.6)$ & 66 (26.6) & $<0.001$ \\
\hline Treated hypercholesterolaemia (n, \%) & $88(27.5)$ & $52(27.4)$ & 0.974 \\
\hline Treated hypertension (n, \%) & 268 (62.9) & $166(66.4)$ & 0.361 \\
\hline Myocardial infarction (n, \%) & $67(15.0)$ & 65 (25.3) & 0.001 \\
\hline Angina pectoris (n, \%) & $150(33.6)$ & $112(42.9)$ & 0.014 \\
\hline Congestive heart failure (n, \%) & $16(3.6)$ & $32(12.5)$ & $<0.001$ \\
\hline Prior PTCA (\%) & $22(4.9)$ & $17(6.5)$ & 0.373 \\
\hline Prior CABG (\%) & $6(1.3)$ & $6(2.3)$ & 0.343 \\
\hline \multicolumn{4}{|l|}{ Killip class (n, \%) } \\
\hline 1 & $144(32.1)$ & $91(34.7)$ & \multirow[t]{4}{*}{0.299} \\
\hline II & $283(63.2)$ & $154(58.8)$ & \\
\hline III & $19(4.2)$ & $17(6.5)$ & \\
\hline IV & $2(0.4)$ & $0(0.0)$ & \\
\hline \multicolumn{4}{|l|}{ Infarct location (n, \%) } \\
\hline Anterior & $212(47.3)$ & $167(64.0)$ & \multirow[t]{5}{*}{$<0.001$} \\
\hline Posterior & $38(8.5)$ & $15(5.7)$ & \\
\hline Lateral & $35(7.8)$ & $16(6.1)$ & \\
\hline Inferoposterior & 109 (24.3) & $28(10.7)$ & \\
\hline Others & 54 (12.1) & 35 (13.4) & \\
\hline PTCA performed at entry ( $n, \%)$ & $149(33.3)$ & $62(23.8)$ & 0.008 \\
\hline Thrombolytic therapy performed at entry $(n, \%)$ & $171(38.2)$ & $101(38.5)$ & 0.920 \\
\hline \multicolumn{4}{|l|}{ Type of thrombolytic therapy (n, \%) } \\
\hline Streptokinase & $69(40.4)$ & $46(45.5)$ & \multirow[t]{5}{*}{0.234} \\
\hline Alteplase & $29(17.0)$ & $10(9.9)$ & \\
\hline Tenecteplase & 47 (27.5) & $30(29.7)$ & \\
\hline Reteplase & $7(4.1)$ & $8(7.9)$ & \\
\hline Other & $19(11.1)$ & $7(6.9)$ & \\
\hline Estimated GFR (mL/min, mean \pm SD) & $89.4 \pm 34.3$ & $82.9 \pm 30.5$ & 0.015 \\
\hline NT-proBNP (pg/mL, mean $\pm S D)$ & $1601 \pm 2485$ & $2809 \pm 3896$ & $<0.001$ \\
\hline $\mathrm{SBP}(\mathrm{mm} \mathrm{Hg}, \operatorname{mean} \pm \mathrm{SD})$ & $139.3 \pm 23.4$ & $140.8 \pm 225.0$ & 0.433 \\
\hline $\mathrm{DBP}(\mathrm{mm} \mathrm{Hg}, \mathrm{mean} \pm \mathrm{SD})$ & $82.4 \pm 13.5$ & $83.9 \pm 13.7$ & 0.155 \\
\hline $\mathrm{HR}(\mathrm{bpm}, \mathrm{mean} \pm \mathrm{SD})$ & $78.0 \pm 15.6$ & $82.5 \pm 17.9$ & $<0.001$ \\
\hline
\end{tabular}

The interaction test for the primary outcome measure between the two study subgroups was not statistically significant and returned an OR of $1.28(0.67$ to 2.47$)$ for the $40 \%$ cut-off $(\mathrm{p}=0.451)$ and an OR of 1.29 (0.47 to $3.53)$ for the $50 \%$ cut-off $(\mathrm{p}=0.615)$.

In general, zofenopril tended to be more effective than ramipril at increasing levels of LVEF, though a statistically significant difference between the two treatments was observed only for the subgroup with an LVEF between $40 \%$ and $50 \%$ (figure 2).

\section{Secondary outcome measures}

During the 1-year observation, the cumulative incidence of cardiovascular deaths was small in both study subgroups, and differences between treatments did not achieve statistical significance (table 2). A statistically significant $(p=0.013)$ difference in the rate of hospital admission for cardiovascular causes was observed between zofenopril and ramipril in the subset of patients with preserved LVEF (table 2). Conversely, the risk of hospitalisation did not significantly differ $(p=0.255)$ in zofenopril-treated and ramipril-treated patients with impaired LVEF (table 2).

When an LVEF cut-off of $50 \%$ was applied for selection of study subgroups, cardiovascular deaths occurred only in the subgroup of patients with an LVEF $\leq 50 \%$ (OR zofenopril vs ramipril: 1.50 (0.69 to 3.25), $\mathrm{p}=0.305$ ). Overall, 10 of 40 patients with an LVEF $>50 \%$ 
Table 2 Absolute and relative frequency (\%) of causes of cardiovascular death and of major cardiovascular events requiring hospitalisation in patients with a preserved left ventricular ejection fraction (LVEF) $>40 \%$ and with an impaired LVEF $(\leq 40 \%)$ randomised to zofenopril or ramipril

\begin{tabular}{|c|c|c|c|c|}
\hline & \multicolumn{2}{|c|}{ Preserved LVEF } & \multicolumn{2}{|c|}{ Impaired LVEF } \\
\hline & $\begin{array}{l}\text { Zofenopril } \\
(n=213) \\
n(\%)\end{array}$ & $\begin{array}{l}\text { Ramipril } \\
(n=235) \\
n(\%) \\
\end{array}$ & $\begin{array}{l}\text { Zofenopril } \\
(n=151) \\
n(\%)\end{array}$ & $\begin{array}{l}\text { Ramipril } \\
(\mathrm{n}=111) \\
\mathrm{n}(\%)\end{array}$ \\
\hline \multicolumn{5}{|l|}{ Major cardiovascular events (death or hospitalisation) } \\
\hline Death due to congestive heart failure & $2(0.9)$ & $1(0.4)$ & $2(1.3)$ & $1(0.9)$ \\
\hline Death due to acute myocardial infarction & - & $1(0.4)$ & $6(3.9)$ & - \\
\hline Sudden death & $1(0.4)$ & $1(0.4)$ & 5 (3.3) & $5(4.5)$ \\
\hline Death due to cardiac rupture & - & - & $1(0.6)$ & $1(0.9)$ \\
\hline Death due to stroke & - & - & - & $1(0.9)$ \\
\hline Hospitalisation due to congestive heart failure & $2(0.9)$ & $3(1.2)$ & $2(1.3)$ & $4(3.6)$ \\
\hline Hospitalisation due to acute myocardial infarction & $5(2.3)$ & $9(3.8)$ & $8(5.2)$ & $7(6.3)$ \\
\hline Hospitalisation due to angina pectoris & $13(6.1)$ & $14(5.9)$ & $7(4.6)$ & $8(7.2)$ \\
\hline Hospitalisation due to decline in LVEF >15\% & $7(3.2)$ & $18(7.6)$ & $8(5.2)$ & $10(9.0)$ \\
\hline Hospitalisation due to revascularisation & $13(6.1)$ & $22(9.3)$ & $12(7.9)$ & $8(7.2)$ \\
\hline Hospitalisation due to other causes & $5(2.3)$ & $8(3.4)$ & $6(3.9)$ & $4(3.6)$ \\
\hline $\begin{array}{l}\text { All causes of cardiovascular death or } \\
\text { hospitalisation }\end{array}$ & $48(22.5)$ & $77(32.8)$ & $57(37.7)$ & $49(44.1)$ \\
\hline $\begin{array}{l}\text { OR }(95 \% \mathrm{Cl}) \text { for } 1 \text {-year cardiovascular mortality } \\
\text { or hospitalisation }\end{array}$ & \multicolumn{2}{|c|}{0.59 (0.39 to 0.91$)$} & \multicolumn{2}{|c|}{$0.81(0.48$ to 1.34$)$} \\
\hline p Value & 0.015 & & 0.403 & \\
\hline \multicolumn{5}{|l|}{ Cardiovascular death } \\
\hline Congestive heart failure & $2(0.9)$ & $1(0.4)$ & $2(1.3)$ & $1(0.9)$ \\
\hline Acute myocardial infarction & - & $1(0.4)$ & $6(3.9)$ & - \\
\hline Sudden death & $1(0.4)$ & $1(0.4)$ & 5 (3.3) & $5(4.5)$ \\
\hline Cardiac rupture & - & - & $1(0.6)$ & $1(0.9)$ \\
\hline Stroke & - & - & - & $1(0.9)$ \\
\hline All causes of cardiovascular death & $3(1.4)$ & $3(1.3)$ & $14(9.3)$ & $8(7.2)$ \\
\hline OR $(95 \% \mathrm{Cl})$ for 1 -year cardiovascular mortality & \multicolumn{2}{|c|}{1.11 (0.22 to 5.53) } & \multicolumn{2}{|c|}{$1.36(0.54$ to 3.41$)$} \\
\hline $\mathrm{p}$ Value & \multicolumn{2}{|c|}{0.904} & \multicolumn{2}{|c|}{0.523} \\
\hline \multicolumn{5}{|c|}{ Major cardiovascular events requiring hospitalisation } \\
\hline Congestive heart failure & $2(0.9)$ & $3(1.2)$ & $2(1.3)$ & $4(3.6)$ \\
\hline Acute myocardial infarction & 5 (2.3) & $9(3.8)$ & $8(5.2)$ & $7(6.3)$ \\
\hline Angina pectoris & $13(6.1)$ & $14(5.9)$ & $7(4.6)$ & $8(7.2)$ \\
\hline Decline in LVEF >15\% & $7(3.2)$ & $18(7.6)$ & $8(5.2)$ & $10(9.0)$ \\
\hline Revascularisation & $13(6.1)$ & $22(9.3)$ & $12(7.9)$ & $8(7.2)$ \\
\hline Other causes & 5 (2.3) & 8 (3.4) & $6(3.9)$ & $4(3.6)$ \\
\hline $\begin{array}{l}\text { All causes of major cardiovascular events requiring } \\
\text { hospitalisation }\end{array}$ & $45(21.4)$ & 74 (31.9) & $43(31.4)$ & $41(39.8)$ \\
\hline $\begin{array}{l}\text { OR }(95 \% \mathrm{Cl}) \text { for } 1 \text {-year cardiovascular } \\
\text { hospitalisation }\end{array}$ & \multicolumn{2}{|c|}{0.58 (0.38 to 0.89) } & \multicolumn{2}{|c|}{$0.73(0.43$ to 1.26$)$} \\
\hline $\mathrm{p}$ Value & \multicolumn{2}{|l|}{0.013} & \multicolumn{2}{|l|}{0.255} \\
\hline
\end{tabular}

treated with zofenopril (25\%) and 16 of 43 treated with ramipril $(37 \%)$ were hospitalised (OR $0.56 \quad(0.22$ to $1.45), \mathrm{p}=0.231)$. In the subgroup with impaired LVEF $(\leq 50 \%)$ the rate of hospitalisation was $25 \%$ (78 of 307 ) under zofenopril and 34\% (99 of 292) under ramipril (OR 0.66 (0.47 to 0.95$), \mathrm{p}=0.023)$.

\section{LVEF values during the study}

Figure 3 shows the average LVEF values measured at baseline and after 1, 6 and 12 months of follow-up in the subgroups of patients with preserved and in those with impaired LVEF, separately for patients treated with zofenopril and ramipril. Baseline LVEF values were significantly $(\mathrm{p}<0.001)$ increased at the end of the 1-year observation by either treatment, in both study subgroups, with no significant differences between zofenopril-treated and ramipril-treated patients. As in the main study, improvement in LVEF (increase $\geq 5 \%$ ) at the end of treatment was achieved by the same proportion of participants in the two treatment arms. However, the rate of improvement was larger in patients with an impaired LVEF (72\% vs $61 \%$ of patients with preserved LVEF, $\mathrm{p}=0.006$ ).

\section{DISCUSSION}

Our retrospective analysis of the SMILE-4 Study suggests that patients with post-AMI with preserved LVEF may gain 

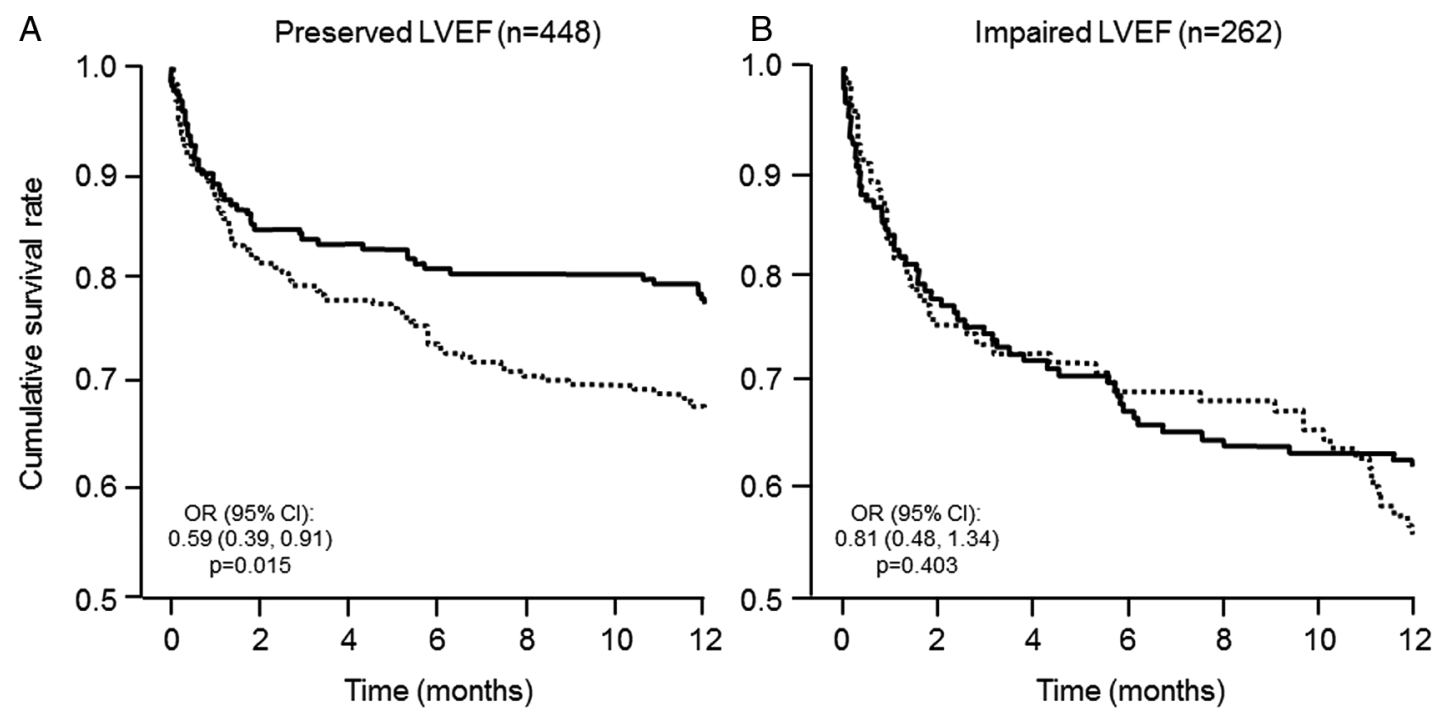

Number at risk

$\begin{array}{llllllll}\text { Zofenopril } & 213 & 180 & 177 & 172 & 171 & 171 & 166 \\ \text { Ramipril } & 235 & 191 & 183 & 173 & 166 & 164 & 159\end{array}$

$\begin{array}{lllllll}151 & 118 & 109 & 102 & 98 & 96 & 95 \\ 111 & 84 & 81 & 77 & 76 & 73 & 63\end{array}$

Figure 1 Cumulative survival without events during 1 year in patients with preserved systolic function (left ventricular ejection fraction (LVEF) $>40 \%$ ) and with impaired systolic function (LVEF $\leq 40 \%$ ) treated with zofenopril (continuous line) or ramipril (dotted line).

a larger clinical benefit from treatment with zofenopril than with ramipril, resulting in a more effective prevention of major cardiovascular outcomes. To the best of our knowledge, this is the first evidence derived from a direct comparative trial that treating patients with $\mathrm{HF}$ at relatively lower risk, with two different ACE inhibitors, may affect patients' prognosis to a significantly different extent.

The larger reduction in the combined risk of cardiovascular morbidity and mortality observed in zofenopril- treated compared to ramipril-treated patients may be explained by examining the specific pharmacological properties of the two drugs. As suggested in the past, due to the central role of tissue ACE in endothelial function, ACE inhibitors may possess anti-ischaemic effects, though differences in the effect may be observed among the various representatives of this class of drugs. ${ }^{16}$ Experimental models and studies in patients with hypertension support the hypothesis that sulfhydryl-containing

1-year cardiovascular mortality and hospitalisation for cardiovascular causes by LVEF

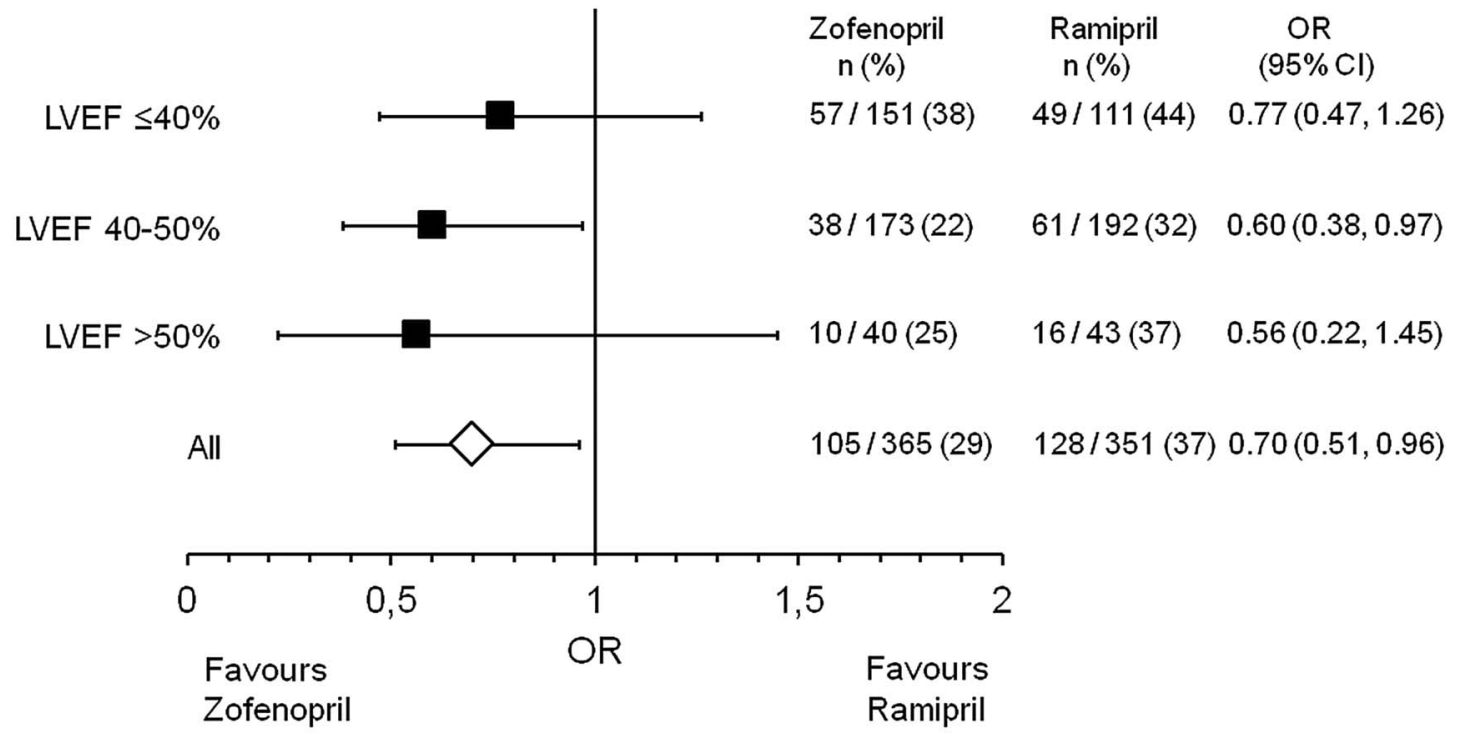

Figure $2 \mathrm{OR}$ and $95 \% \mathrm{Cl}$ for 1-year combined occurrence of cardiovascular mortality or hospitalisation for cardiovascular causes according to ranges of left ventricular ejection fraction (LVEF). 

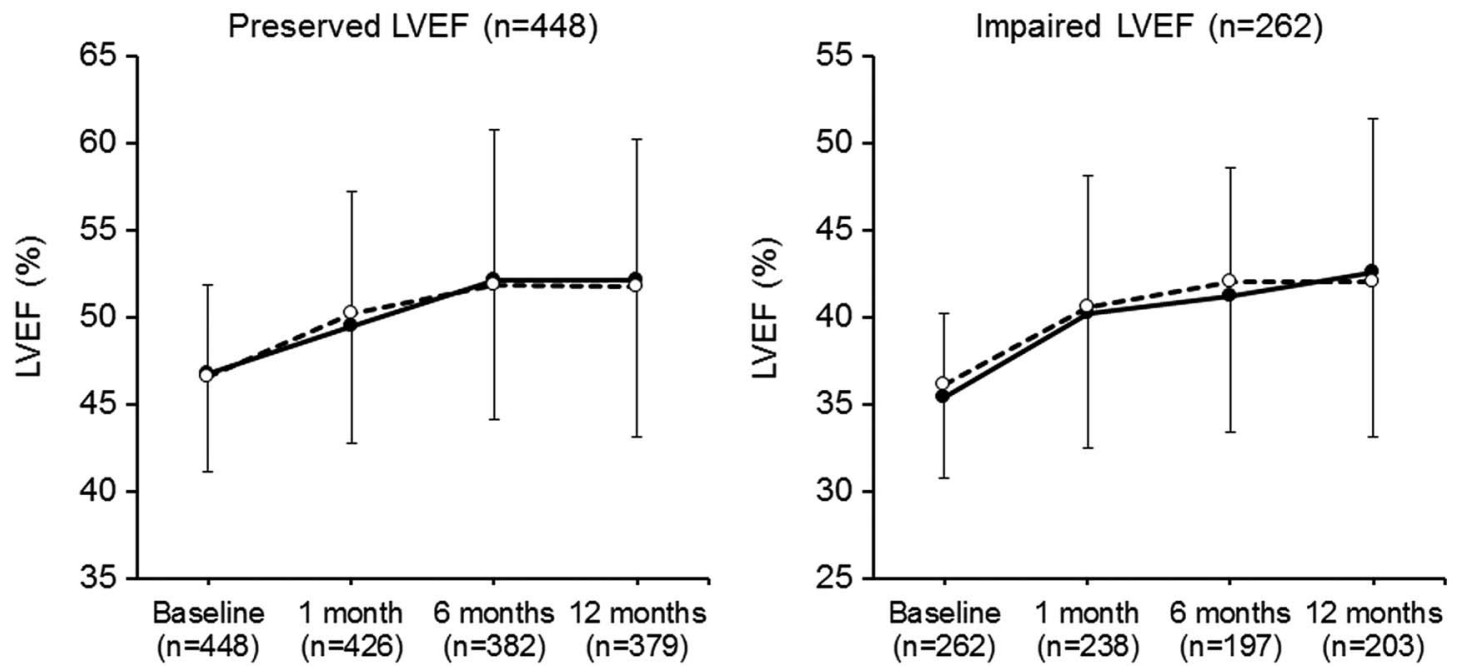

Figure 3 Average left ventricular ejection fraction (LVEF) values during the 1 year of observation in patients with preserved systolic function (LVEF $>40 \%$ ) and with impaired systolic function (LVEF $\leq 40 \%$ ) treated with zofenopril (continuous line) and ramipril (dotted line).

ACE inhibitors, such as captopril or zofenopril, may have specific cardioprotective features. ${ }^{17-19}$ In humans, long-term treatment with zofenopril has been associated with a reduction in oxidative stress and an improvement in the nitric oxide pathway, with consequent superior vasculoprotective activity as compared to the nonsulfhydryl ACE inhibitor enalapril. ${ }^{20}{ }^{21}$ It has been postulated that the antioxidant protection of zofenopril observed in all these studies could be related to the high scavenging activity of free radicals mediated by the sulfhydryl moiety of the drug. ${ }^{22}$ This may turn into a clinically important anti-ischaemic effect, as suggested by the results of the SMILE-ISCHEMIA Study. ${ }^{23}$

Additional interesting results of our analysis worth discussing: First, in the whole study population, including the subgroup of patients with a preserved LVEF, at variance from ramipril, the clinical efficacy of zofenopril did not appear to be counteracted by the concomitant administration of $\mathrm{ASA},{ }^{13}$ confirming evidence from previous studies. $^{24-26}$

Second, our results add a new source of evidence to address the question of whether treatment with ACE inhibitors may have a positive impact on long-term outcomes of patients with stable coronary artery disease who have preserved LVEF. In previous randomised, placebo-controlled trials, different ACE inhibitors (enalapril, perindopril, ramipril or trandolapril) significantly lowered the risk of hospitalisation for HF (from $17 \%$ to $24 \%$, depending on the studies) and of the combined end point of cardiovascular mortality and hospitalisation for $\mathrm{HF}$ (from $15 \%$ to $26 \%$ ). ${ }^{8}{ }^{9}$ However, to date, most studies failed to show a clear improvement in mortality during prolonged follow-up with an ACE inhibitor, with risk reduction ranging from $10 \%$ to $15 \%$ for total mortality and from $14 \%$ to $19 \%$ for cardiovascular mortality. ${ }^{7-9}$ In our study, zofenopril reduced the risk of fatal and non-fatal cardiovascular outcomes, with respect to ramipril, by $40 \%$, indicating a larger effect than that observed in previous placebo-controlled studies in patients with HF and preserved LVEF. However, most of the benefit of zofenopril on cardiovascular outcomes was driven by a reduction in the rate of hospitalisation by $52 \%$; zofenopril did not show any additional benefit to that of ramipril in terms of prevention of cardiovascular death, though the number of deaths was very small during the study in the subgroup of patients with preserved LVEF. This strengthens the hypothesis that efficacy of zofenopril in patients with $\mathrm{HF}$ with no echocardiographic signs of LVD may differ from that of the other previously studied ACE inhibitors, and confirms that the number of patients needed to treat in order to prevent any additional death with an ACE inhibitor is qualitatively different in patients with preserved LVEF and in those with impaired LVEF. ${ }^{27}$

Finally, we observed a significant and similar improvement in LVEF with both drugs, irrespective of the subset of patients. However, the magnitude of the improvement was larger in patients with impaired LVEF, confirming the positive effect of ACE inhibition on cardiac remodelling and haemodynamics in cases of LVD. In the group of patients with preserved LVEF, the lack of difference in LVEF improvement between the two study treatment arms, in spite of a superior clinical efficacy of zofenopril, may support the hypothesis that zofenopril may affect patients' prognosis independently of any haemodynamic effect.

\section{Study limitations}

Some limitations of our study also deserve to be discussed. First, the study is based on a post hoc analysis of the SMILE-4 Study, with a relatively small case number resulting from splitting the population into four groups, and might be underpowered to yield any meaningful conclusion, as the event rate is too low in the different subgroups. As a matter of fact, post hoc power 
calculation for the primary study end point revealed a power of $68 \%$ for the group with preserved LVEF $(>40 \%)$ and of only $18 \%$ for that with impaired LVEF $(\leq 40 \%)$. Nonetheless, the prevalence of HF with preserved LVEF in our set of participants was comparable with that reported in epidemiological studies including patients with stable ischaemic heart disease $(63 \%$ vs 50 $60 \%$ ), suggesting that our sample may be representative of the general population. ${ }^{28-30}$ Second, this post hoc analysis was retrospective. However, since no prospective comparative studies of two ACE inhibitors in patients with post-AMI with HF and preserved LVEF are available, we must rely on evidence provided by these retrospective data, which are the only data available for the analysis. Third, at variance from previous studies in patients with preserved LVEF, our study did not make use of a placebo control and thus comparison with and inference from existing published data may be difficult. Finally, several characteristics of the patients with preserved LVEF in our study are quite atypical and in contrast with previous findings. ${ }^{30}$ In particular, our patients tended to be younger with a lower prevalence of diabetes than the cohort with impaired LVEF, while the opposite has been previously shown. ${ }^{30}$ Additionally, hypertension history was not different between the two groups, while patients with preserved LVEF are usually more likely to display high blood pressure values. ${ }^{30}$ Despite the relatively young age of our sample, the proportion of primary percutaneous interventions and of patients receiving thrombolysis therapy was incredibly low (30\% and $38 \%$ of the patients, respectively), which might not be representative of current practice.

\section{Conclusions}

Our retrospective analysis suggests that zofenopril is more effective than ramipril in reducing cardiovascular hospitalisation in patients with post-AMI with an LVEF $>40 \%$, even in presence of concomitant therapy with ASA. This provides further support for the treatment of a wide range of patients with post-AMI with an effective ACE inhibitor.

\author{
Author affiliations \\ ${ }^{1}$ Unit of Internal Medicine, Policlinico S. Orsola, University of Bologna, \\ Bologna, Italy \\ ${ }^{2}$ Italian Institute of Telemedicine, Varese, Italy \\ ${ }^{3}$ Division of Cardiology, University of Palermo, Palermo, Italy \\ ${ }^{4}$ University and Emergency Hospital, Bucharest, Romania \\ ${ }^{5}$ Division of Cardiology, University of Perugia, Perugia, Italy
}

Twitter Follow Stefano Omboni at @iitelemed

Contributors SO drafted the work; $\mathrm{CB}$ revised it critically for important intellectual content; $\mathrm{CB}$ and $\mathrm{SO}$ were responsible for the final approval of the version to be published.

Funding This work was financially supported by Menarini International Operations Luxembourg SA; Laboratori Guidotti SpA and Istituto Lusofarmaco d'Italia S.p.A. through an unconditional and unrestricted grant. The funding source did not influence or commented on planned methods, protocol, data analysis and the draft report.
Competing interests CB: Abbott USA shareholder, BMS USA shareholder, Boheringer Ingelheim consultancy, Menarini International Consultancy. SO has received a grant for manuscript preparation from Menarini International.

Patient consent Obtained.

Ethics approval Institutional Review Board of the Policlinico S Orsola, Bologna.

Provenance and peer review Not commissioned; externally peer reviewed.

Open Access This is an Open Access article distributed in accordance with the Creative Commons Attribution Non Commercial (CC BY-NC 4.0) license, which permits others to distribute, remix, adapt, build upon this work noncommercially, and license their derivative works on different terms, provided the original work is properly cited and the use is non-commercial. See: http:// creativecommons.org/licenses/by-nc/4.0/

\section{REFERENCES}

1. Flather MD, Yusuf S, Køber L, et al. Long-term ACE-inhibitor therapy in patients with heart failure or left-ventricular dysfunction: a systematic overview of data from individual patients. Lancet 2000;355:1575-81.

2. [No authors listed]. Indications for ACE inhibitors in the early treatment of acute myocardial infarction: systematic overview of individual data from 100,000 patients in randomized trials. Circulation 1998;97:2202-12

3. Steg PG, James SK, Atar D, et al. Task Force on the management of ST-segment elevation acute myocardial infarction of the European Society of Cardiology (ESC). ESC Guidelines for the management of acute myocardial infarction in patients presenting with ST-segment elevation. Eur Heart J 2012;33:2569-619.

4. O'Gara PT, Kushner FG, Ascheim DD, et al. American College of Cardiology Foundation/American Heart Association Task Force on Practice Guidelines. 2013 ACCF/AHA guideline for the management of ST-elevation myocardial infarction: a report of the American College of Cardiology Foundation/American Heart Association Task Force on Practice Guidelines. Circulation 2013;127:e362-425.

5. Finn SD, Gardin JM, Abrams J, et al. American College of Cardiology Foundation/American Heart Association Task Force. 2012 ACCF/AHA/ACP/AATS/PCNA/SCAI/STS guideline for the diagnosis and management of patients with stable ischemic heart disease: a report of the American College of Cardiology Foundation/American Heart Association task force on practice guidelines, and the American College of Physicians, American Association for Thoracic Surgery, Preventive Cardiovascular Nurses Association, Society for Cardiovascular Angiography and Interventions, and Society of Thoracic Surgeons. Circulation 2012;126:e354-471.

6. Anderson JL, Adams CD, Antman EM, et al. American College of Cardiology Foundation/American Heart Association Task Force on Practice Guidelines. 2012 ACCF/AHA focused update incorporated into the ACCF/AHA 2007 guidelines for the management of patients with unstable angina/non-ST-elevation myocardial infarction: a report of the American College of Cardiology Foundation/American Heart Association Task Force on Practice Guidelines. Circulation 2013;127:e663-828.

7. Al-Mallah $\mathrm{MH}$, Tleyjeh IM, Abdel-Latif AA, et al. Angiotensin-converting enzyme inhibitors in coronary artery disease and preserved left ventricular systolic function. A systematic review and meta-analysis of randomized controlled trials. J Am Coll Cardiol 2006;47:1576-83.

8. Danchin N, Cucherat M, Thuillez C, et al. Angiotensin-converting enzyme inhibitors in patients with coronary artery disease and absence of heart failure or left ventricular systolic dysfunction. An overview of long-term randomized controlled trials. Arch Intern Med 2006;166:787-96.

9. Coleman $\mathrm{Cl}$, Baker WL, Kluger J, et al. Comparative effectiveness of angiotensin converting enzyme inhibitors or angiotensin II receptor blockers added to standard medical therapy for treating stable ischemic heart disease. Rockville, MD: Agency for Healthcare Research and Quality (US), 2009. http://www.ncbi.nlm.nih.gov/ books/NBK36476/

10. Borghi C, Ambrosioni E, Novo S, et al., SMILE-4 Working Party. Comparison between zofenopril and ramipril in combination with acetylsalicylic acid in patients with left ventricular systolic dysfunction after acute myocardial infarction: results of a randomized, double-blind, parallel-group, multicenter, European study (SMILE-4). Clin Cardiol 2012;35:416-23. 
11. Ambrosioni E, Borghi C, Magnani B, The Survival of Myocardial Infarction Long-Term Evaluation (SMILE) Study Investigators. The effect of the angiotensin-converting-enzyme inhibitor zofenopril on mortality and morbidity after anterior myocardial infarction. $N$ Engl $J$ Med 1995;332:80-5.

12. Lang RM, Bierig M, Devereux RB, et al. Chamber Quantification Writing Group; American Society of Echocardiography's Guidelines and Standards Committee; European Association of Echocardiography. Recommendations for chamber quantification: a report from the American Society of Echocardiography's Guidelines and Standards Committee and the Chamber Quantification Writing Group, developed in conjunction with the European Association of Echocardiography, a branch of the European Society of Cardiology. J Am Soc Echocardiogr 2005;18:1440-63.

13. McMurray JJ, Adamopoulos S, Anker SD, et al. ESC Committee for Practice Guidelines. ESC Guidelines for the diagnosis and treatment of acute and chronic heart failure 2012: The Task Force for the Diagnosis and Treatment of Acute and Chronic Heart Failure 2012 of the European Society of Cardiology. Developed in collaboration with the Heart Failure Association (HFA) of the ESC. Eur Heart $J$ 2012;33:1787-847.

14. Redfield MM, Chen HH, Borlaug BA, et al. RELAX Trial. Effect of phosphodiesterase- 5 inhibition on exercise capacity and clinical status in heart failure with preserved ejection fraction: a randomized clinical trial. JAMA 2013;309:1268-77.

15. Lam CS, Roger VL, Rodeheffer RJ, et al. Pulmonary hypertension in heart failure with preserved ejection fraction: a community-based study. J Am Coll Cardiol 2009;53:1119-26.

16. Ertl G, Hu K. Anti-ischemic potential of drugs related to the reninangiotensin system. J Cardiovasc Pharmacol 2001;37(Suppl 1): S11-20.

17. Frascarelli S, Ghelardoni S, Ronca-Testoni S, et al. Cardioprotective effect of zofenopril in perfused rat heart subjected to ischemia and reperfusion. J Cardiovasc Pharmacol 2004:43:294-9.

18. Napoli C, Cicala C, D'Armiento FP, et al. Beneficial effects of ACE-inhibition with zofenopril on plaque formation and low-density lipoprotein oxidation in Watanabe heritable hyperlipidemic rabbits. Gen Pharmacol 1999;33:467-77.

19. Evangelista S, Manzini S. Antioxidant and cardioprotective properties of the sulphydryl angiotensin-converting enzyme inhibitor zofenopril. J Int Med Res 2005;33:42-54.

20. Napoli C, Sica V, de Nigris F, et al. Sulfhydryl angiotensin-converting enzyme inhibition induces sustained reduction of systemic oxidative stress and improves the nitric oxide pathway in patients with essential hypertension. Am Heart J 2004;148:e5.
21. Pasini AF, Garbin U, Nava MC, et al. Effect of sulfhydryl and non-sulfhydryl angiotensin-converting enzyme inhibitors on endothelial function in essential hypertensive patients. $\mathrm{Am} \mathrm{J}$ Hypertens 2007;20:443-50.

22. Chopra M, Beswick H, Clapperton M, et al. Antioxidant effects of angiotensin-converting enzyme (ACE) inhibitors: free radical and oxidant scavenging are sulfhydryl dependent, but lipid peroxidation is inhibited by both sulfhydryl- and nonsulfhydryl-containing ACE inhibitors. J Cardiovasc Pharmacol 1992;19:330-40.

23. Borghi C, Ambrosioni E, on behalf of the Survival of Myocardial Infarction Long-term Evaluation (SMILE) Study Group. Effects of zofenopril on myocardial ischemia in post-myocardial infarction patients with preserved left ventricular function: the Survival of Myocardial Infarction Long-term Evaluation (SMILE)-ISCHEMIA study. Am Heart J 2007;153:e7-14.

24. Przyklenk K, Kloner RA. Angiotensin converting enzyme inhibitors improve contractile function of stunned myocardium by different mechanisms of action. Am Heart $J$ 1991;121:1319-30.

25. Zhu BQ, Sievers RE, Browne AE, et al. Comparative effects of aspirin with ACE inhibitor or angiotensin receptor blocker on myocardial infarction and vascular function. $J$ Renin Angiotensin Aldosterone Syst 2003;4:31-7.

26. Ehring T, Baumgart D, Krajcar M, et al. Attenuation of myocardial stunning by the ACE inhibitor ramiprilat through a signal cascade of bradykinin and prostaglandins but not nitric oxide. Circulation 1994;90:1368-85.

27. White CM, Greene L. Summary of AHRQ's comparative effectiveness review of angiotensin-converting enzyme inhibitors or angiotensin II receptor blockers added to standard medical therapy for treating stable ischemic heart disease. J Manag Care Pharm 2011;17(Suppl 5):S1-15.

28. Vivo RP, Krim SR, Krim NR, et al. Care and outcomes of Hispanic patients admitted with heart failure with preserved or reduced ejection fraction: findings from get with the guidelines-heart failure. Circ Heart Fail 2012;5:167-75.

29. Magaña-Serrano JA, Almahmeed W, Gomez E, et al., I PREFER Investigators. Prevalence of heart failure with preserved ejection fraction in Latin American, Middle Eastern, and North African Regions in the I PREFER study (Identification of Patients With Heart Failure and PREserved Systolic Function: an epidemiological regional study). Am J Cardiol 2011;108:1289-96.

30. Owan TE, Hodge DO, Herges RM, et al. Trends in prevalence and outcome of heart failure with preserved ejection fraction. $N$ Engl $J$ Med 2006;355:251-9. 